\title{
ESPACIOS BIOGEOGRÁFICOS DEL EXTREMO SUR DEL PERÚ
}

\author{
José Pizarro Neyra ${ }^{\prime}$
}

\author{
$R E S U M E N$
}

\begin{abstract}
En el presente trabajo se expone la distribución geográfica de ciertos organismos en el extremo sur del Perú, especialmente en las zonas de costa y serranía esteparia.

Se abordan cuestiones relacionadas con la influencia de los factores abióticos y bióticos sobre la distribución de la fauna y flora, señalando los endemismos, y centros de origen.

Se propone que ésta región es importante, desde el punto de vista evolutivo y biogeográfico, en recursos florísticos y faunísticos, para los cuales se deben de priorizar medidas de conservación en el extremo sur del Perú.
\end{abstract}

\section{A B S TR A C T}

This work is to explain geographical distribution of some organisms in the far south of Peru and neighboring region especially coastal desert and low highlands.

Endemisms, origin centres and lack of information about the biotic resources at this region and the influence of biotic and abiotic factors on distribution and abundance of fauna and flora are offered in this paper.

Our proposition is the conservation of the natural resources in the far south Peru which are biogeographical and evolutionary important.

\section{INTRODUCCIÓN}

El extremo sur del Perú ha sido identificada por INRENA (1994) como unárea en la que se desconocen la diversidad de algunos grupos de fauna y flora. Ello nos lleva a documentar una alternativa de bioregionalización con el fin de sistematizar la información que pueda servir para la conservación de los recursos bióticos de la zona.

Esquemáticamente, los territorios del sur del Perú se encuentran ubicados en la región neotropical, sugerida por Wallace y Sclater; como una región particular del mundo en base principalmente a patrones de distribución de fauna (Macarthur, 1972).
La región neotropical se caracteriza por estar ubicada entre los trópicos de Cáncer y Capricornio o alrededor de éstos. Cabrera \& Willink (1980), en un trabajo clásico, determinaron la existencia de cinco dominios dentro de la región neotropical, de los cuales Tacna y el extremo sur del Perú se encuentran incluidos en las provincias : Puneña, Desértisa y Altoandina. En la Región oceánica estos autores mencionan al dominio oceánico peruano chileno que se encuentra frente a nuestras costas. Brack-Egg (1985) sugirió la clasificación biogeográfica del Perú en base a ecorregiones, de las cuales el territorio de Tacna y

1 Miembro del Centro de Estudios e Investigación del Medio Ambiente. 
Moquegua tendrían las siguientes: Mar frío de la corriente de Humboldt, Desierto costero, Serranía esteparia y Puna. Este será el enfoque utilizado para analizar la biogeografía de la zona, agrupando a la fauna por grupo taxonómico y a la flora en comunidades vegetales.

\section{LOS ESPACIOS BIOGEOGRÁFICOS DEL SUR Y ALREDEDORES}

Hemos definido el espacio biogeográfico como una entidad no rígida que expresa la distribución de organismos en un territorio, tanto como su función dentro de ecosistemas y su importancia evolutiva. Así los espacios geográficos son provincias biogeográficas, centros de origen o evolución de especies y áreas de importancia para la conservación.

Hay que aclarar que en los términos de "extremo sur del Perú" dejamos entreveer que las características ecológicas de Tacna Moquegua y zonas de puna árida de Puno son las mismas que las zonas contiguas de Chile y Bolivia, por lo que haremos continuas alusiones a estos territorios.

\section{Antecedentes}

Desde el punto de vista climático, la ONERN (1986) considera que aproximadamente desde $17^{\circ}$ Lat. Sur nos encontramos en la región latitudinal templado cálida. Una identificación de tipos climáticos en base a la clasificación de Koppen, propone que en nuestra región existen: clima semicálido muy seco; clima templado subhúmedo; clima de valles mesoandinos; clima de Tundra y clima gélido.

En una revisión rápida del sistema de clasificación de suelos, encontraremos que el territorio de Tacna presenta suelos de tipo yermosólico, lítico y andosólico. Mientras que la clasificación de zonas geomorfológicas del Perú, ofrecida por Bellido (1990), nos permite declarar que en el territorio de Tacna, Moquegua y zonas aledañas del Este y Sur existen para el mar territorial: margen continental, plataforma continental, talud continental, fosas marinas y fondos abisales del Océano Pacífico; mientras que para la zona continental se presentan: linea de costa, cordillera de la costa, llanura costanera, cordillera occidental, arco de volcanes Parinacochas-Barroso, altiplano, depresión altiplánica y cuenca del Titicaca, además de las áreas glaciadas y nevados. La fosa oceánica frente a Tacna y Arica alcanza profundidades de más de 6000 metros.

\section{Influencia del clima y la geomorfología en la distribución de organismos.}

La fosa oceánica llega a ser diferencialmente más profunda frente a las costas de Moquegua, Tacna y Arica que más al sur, en Arequipa, ya que alcanzan una profundidad de 6652 metros según Morris \& Panty (1994). En estas profundidades, en el límite de máxima depleción de oxígeno en el mar abisal, se encuentran poblaciones considerables de bacterias filamentosas, las cuales degradan el material sulfurado orgánico. Otros organismos característicos son los decápodos abisales gigantes, comunes a otras zonas del ambiente submarino, en especial de la familia Lithodidae.

Desde un punto de vista biogeográfico es importante la configuración del relieve para diferenciar el extremo sur de otras zonas de los andes centrales. Gómez-Molina \& Little (1981), quienes mencionan que la máxima amplitud de los andes se alcanza a la altura de Bolivia, da lugar a un espacio geográfico particular: el altiplano y la cuenca del Lago Titicaca, que configuran de por sí una provincia biogeográfica aparte que ha dado lugar a especies endémicas de peces de los géneros Trichomycterus y Orestias además de otros organismos como la pseudorana Telmatobius, siendo un caso típico de especiación alopátrica.

Otra diferencia geomorfológica importante se dá entre la zona costera del extremo sur y parte de la zona norte de Chile. Refiere la existencia de una planicie costera alta y ancha a la altura de Antofagasta (aproximadamente $24^{\circ}$ Lat. Sur); mientras que en Moquegua, Tacna y Arica esta planicie no alcanza altitud y no es ancha, dando lugar a otro distrito de la provincia del desierto costero y que no entraría en la clasificación ni de Brack-Egg, ni de Cabrera \& Willink. La diferencia del gradiente de humedad que disminuye de Norte a Sur ha permitido la evolución de algunas especies distintas de flora y fauna. En la flora son un buen ejemplo Prosopis tamarugo y Tessaria absinthioides, oriundas de la pampa alta costera de Iquique, mientras que en la costa de Tacna se halla Tessaria integrifolia, y en serranía esteparia de Arequipa se halló Prosopis pallida. Los suelos de las pampas costeras presentan costras salinas denominadas "caliche", a los cuales están bien adaptadas las plantas del desierto atacameño, 
mientras que nuestras especies suelen vivir cerca de fuentes de agua dulce (canales, acequias y rios). Esto se correlaciona con las afirmaciones del MOPU (1990), en el sentido de que la vegetación desértica se presenta entre $22^{\circ}$ Lat. sur y $27^{\circ}$ Lat. sur.

Lo que queda demostrado por los trabajos de Giddings (1992) y ampliado por la Southamerica Land Cover Characteristics data base de Estados Unidos, tal como vemos en la Figura 1 .

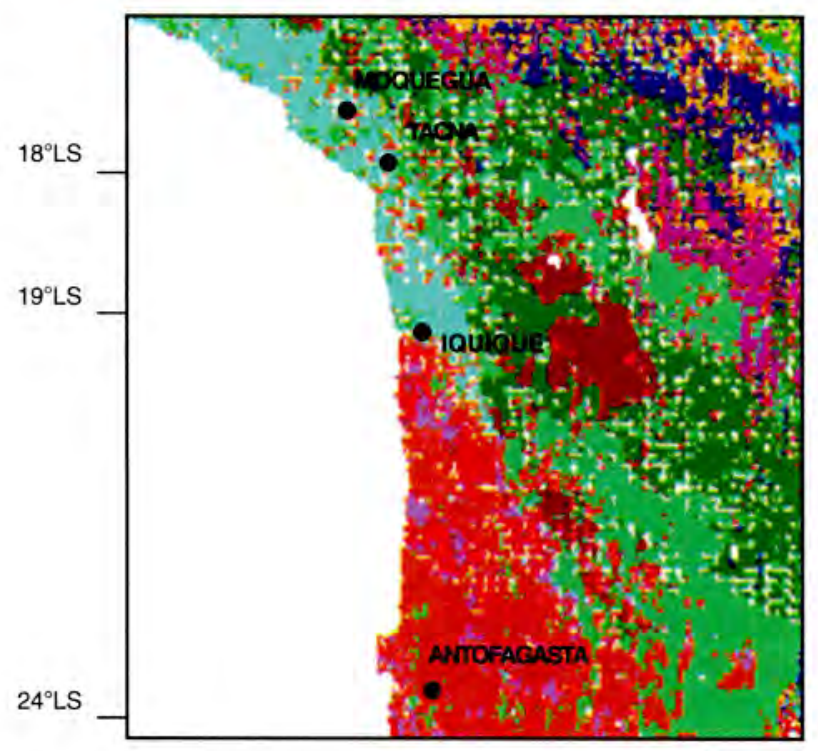

Figura 1. Imagen digitalizada del South America Land Cover Characteristics Database. Las zonas celestes indican vegetación esparcida del desierto costero del Pacífico y las zonas rojas indican muy escasa vegetación o ninguna que comienza alrededor de $19^{\circ} \mathrm{LS}$.

La existencia del arco volcánico que nace en Ayacucho y atravieza esta región hasta Chile, también presenta importancia biogeográfica. Por ejemplo, son comunes a Ayacucho y la precordillera del sur la especie Oreocereus hendriksenianus, presentando una distribución casi continua. El caso de Lobivia es diferente ya que se le considera una especie de distribución discontinua en los andes centrales desde Arequipa y Cusco hasta Bolivia y Chile que utiliza las altas montañas de los andes como si fueran "islas". Un ave, Upucerthia albigula es reportada por Ridgely \& Tudor (1989), con una distribución entre Puquio (Ayacucho) y Putre (Arica). Este ejemplo sirve para explicar la teoría de Vuilleumier (1981), quien con sidera a los andes como una zona donde se presentan gran número de endemismos de aves relacionado con la especiación que sufrieron después que colonizaran las montañas en el período glacial. La colonización provino del Norte, Este y en menor medida del Sur. Un ave llegada del sur, Pterocnemia pennata, hoy encuentra su límite norte de distribución en el altiplano de Moquegua y Puno.

Las especiaciones más espectaculares están dadas por las aves dependientes de bosquetes de Polylepis. En nuestra región existe el antecedente de los bosquetes de Pampa Queñuta (Tacna) y Poma Chico (Tarata) que no han sido estudiados como refugios de fauna, pero Fjeldsa y Kessler (1996) afirman que la especie dominante de Polylepis en Moquegua y Tacna es $P$. rugulosa, una variedad achaparrada de puna que no es común en otros lugares de la sierra.

Los bosquetes altoandinos y bosques de serranía esteparia disminuyen de norte a sur en densidad y esto tiene que ver con la disminución de la gradiente de humedad hacia el sur, que es más árido. Nuestra zona se caracteriza por recibir una tasa de radiación solar encima del promedio mundial, al igual que otras seis zonas del planeta, lo cual provoca mayor evaporación y características de zona árida, donde las precipitaciones son menores a la evaporación.

La gran extensión de los desiertos costeros ha favorecido el aislamiento de las aves. Se reconoce a Arica como límite sur de Thaumastura cora y ello probablemente se deba a la pobreza hídrica de los valles subsiguientes y disminución de la flora melífera. Un mecanismo similar podría operar para explicar los límites de la distribución de Pyrocephalus rubinus y Muscigralla brevicauda en zonas contiguas mencionados por Ridgely \& Tudor (1994).

Finalmente podemos citar como barreras geográficas importantes a las fallas geológicas como Incapuquio (Tacna-Moquegua).

\section{Variación de la biodiversidad en la zona.}

Según Wilson (1994), existe la tendencia a que la diversidad de plantas y animales aumente a medida que se avanza de las regiones polares hacia el Ecuador. Ello implicaría que en nuestra bioregión la diversidad biológica es menor que en unidades biogeográficas situadas inmediatamente al norte del paralelo $17^{\circ}, \mathrm{y}$ superior a la región del extremo desierto que comienza a los $22^{\circ}$ de latitud, aproximadamente. 
En cuanto a la variación de la diversidad con la altura, ésta aumenta con la altitud, de acuerdo con las investigaciones de Kalin et al (1982) en la cordillera de Huaylillas, y de Pearson \& Pearson (1978) en Tacna. Estas investigaciones parecen indicar que existe un aumento de diversidad de especies arriba de los 3500 msnm, empezando a declinar encima de los 4500 msnm.

En ecosistemas particulares hay gran diversidad de ciertas taxa. En los humedales de Ite, por ejemplo, se registran más aves que en ningún otro lugar de la costa. Este lugar es importante por ser el único humedal costero entre Mejía (Arequipa) y Huasco (Chile) (Pizarro y Franco 1994). Algunos ambientes acuáticos altoandinos de la ecorregión Puna presentan gran cantidad de aves, como Loriscota, Vilacota y Vizcachas.

Un aspecto característico de la zona en cuestión es que recibe permanente visita de aves migratorias, las cuales pueden cumplir el papel de diseminador de plantas, lo cual aumenta la diversidad del extremo sur. Se estaría dando el fenómeno de "efecto de borde", en la confluencia de los territorios del desierto de Atacama y los del extremo sur de Perú, que también aumenta la diversidad.

Finalmente, la diferencia entre la diversidad (en especial biodiversidad vegetal) de las vertientes occidentales y orientales de los andes del extremo sur, no está aún esclarecida; pero se dan casos de aislamiento de especies, por ejemplo, Polylepis rugulosa presente exclusivamente en nuestra biorregión, mientras que P. tarapacana y P. besseri están presentes en la cordillera y altiplano de Puno, y zonas adyacentes de Bolivia y Chile. Otro caso conocido es el de Grindelia glutinosa confinada a la vertiente occidental y Grindelia boliviana, ampliamente diseminada en la otra vertiente andina. Estas especies endémicas alrededor de los andes del extremo sur también incluyen ciertos tipos de peces, reptiles y batracios.

\section{ESTRUCTURABIOLÓGICADE LOSESPACIOS BIOGEOGRÁFICOS.}

Se distinguen en estructura por su uniformidad en cuanto a tipo de fauna, flora, variaciones climatológicas, geomorfología y factores antropogénicos.

\section{LAFAUNA}

\section{Dominio Oceánico}

Nos referimos a peces y aves con algunos casos de distribución restringida como Lutra felina, mustélido marino, desde Magallanes (Chile) hasta Lima, en el Perú (Pulido, 1991). También debe considerarse a Phocoena spinipinnis, un cetáceo menor, común en aguas peruanas, pero presente en mayor cantidad que otros en el sur, a decir por los resultados obtenidos por Van Waerebeek et al (1988), y de los hallazgos de osamenta referidos en el litoral de Tacna (Pizarro, 1997).

Otras especies marinas interesantes son las tortugas, las cuales han sido comunes en Tacna, según se desprende de los comentarios de Dagnino (1903). Al respecto, Glade et al (1983) mencionan a Caretta caretta, halladas en mar abierto de la I región de Chile; mientras que Márquez (1990), señala claramente que esta es una tortuga marina de distribución mundial, pero que en las costas occidentales de sudamérica se le halla solamente en dos zonas costeras frente a Arica y Coquimbo (Chile).

La distribución de algunos organismos invertebrados marinos comunes en las costas de Tacna y Moquegua alcanza su límite norte en la costa central. Este es el caso de Pyura chilensis, Concholepas concholepas, Fissurella crassa y Mesodesma donacium.

Las aves ocupan lugar preponderante en éste dominio biogeográfico, siendo en su gran parte migratorias. Un estudio de Morrison et al (1990), demostró que la orilla marina entre Pisagua y Mollendo, la playa arenosa ubicada entre Llostay y La Yarada, presenta una de las mayores densidades de pájaros migratorios de origen neártico en sudamérica y la mayor en el sur del Perú. El litoral de Tacna y Moquegua constituyen el límite sur de distribución de Cinclodes taczanowskii, la única ave passeriforme de orilla marina en el Perú.

\section{Dominio Andino-Patagónico}

Las aves constituyen el grupo faunístico más diverso entre los vertebrados en Tacna. Las aves de distribución restringida a nivel subcontinental y que viven en el extremo sur del Perú y zonas aledañas son: Fulica cornuta, Eulidia yarrellii, Pterocnemia pennata, Xenospingus concolor y Conirostrum tamaruguense. Las aves altoandinas de cuerpos de 
agua son generalmente migratorias y destacan los tres tipos de flamencos Phoenicoparrus andinus, Phoenicoparrus jamesi y Phoenicopterus chilensis, además de la Kiula Nothoprocta ornata y la choca Fulica gigantea, que llega a su límite sur de distribución en la zona altoandina de Tarapacá.

Entre los vertebrados más conspicuos destacan los reptiles, los cuales presentan hasta ocho especies características de Tacna (Carrillo et al, 1995); ellos son : Tropidurus melanopleurus melanopleurus, Microlophus heterolepis, Microlophus quadrivittatus, Phrynosaura stolzmanni, Liolaemus pantherinus, Liolaemus tacnae, Liolaemus alticolor y Phyllodactylus gerrophygus, éste último típico del arenal costero. Sumados a estos saurios debería añadirse a la lista de reptiles de la zona a Phylodrias tachymenoides, ya que Pearson et al (1978) al igual que otros investigadores los reportan para Tacna y Tarata. Phylodrias simonsis, Tachymenis peruviana y Alsophis elegans, son citados por Glade (1993), para la contigua zona de Chile.

Los anfibios de ésta biorregión están poco estudiados, por ello Rodríguez et al (1993), sólo mencionan para Tacna, Moquegua y Arequipa a Bufo arequipensis y para Arequipa y Tacna a Telmatobius peruvianus. Mucha de la biodiversidad de anfibios de Puno y Bolivia puede habitar en nuestra zona.

Entre los roedores podríamos destacar la probable presencia de Chinchilla brevicaudata, en la ecorregión puna del extremo sur del Perú, ya que reportes publicados afirman que en el siglo XIX se exportaba peletería de Chinchilla a través de los puertos del litoral.

Los invertebrados continentales cuentan entre los tipos más importantes a Coleópteros, Dípteros, Odonatos, Orthópteros, Homópteros y Lepidópteros (Insecta); Arácnidos y Acaros (Chelicerata); Miriápodos; entre otros. Son importantes crustáceos y nemátodos en ambientes acuáticos altoandinos, tal como lo han demostrado los estudios de Moreno $Y$ Coronel (1987).

\section{LA FLORA}

\section{Dominio Oceánico}

Franco et al (1995), ha encontrado una flora macrofítica caracterizada por la predominancia de algas rojas en el litoral de Tacna, la que es coincidente en su composición con la de Mollendo y algunos de éstos géneros son comunes en el litoral de Chile, como Corallina. Por otro lado, Gómez (1992) afirma que en aguas del sur del Perú predomina el zooplancton, mientras que en el litoral central y norte (6-15ㄴ. Sur), hasta 60 millas mar adentro, se acentúa la presencia de fitoplancton.

\section{Dominio Andino-Patagónico}

Las comunidades vegetales más importantesihan sido clasificadas en: Lomas, matorral xerofítico, monte ribereño y y humedales costeros (para el desierto costero); laderas y valles andinos, formaciones espinosas (para la serranía esteparia) y pajonales, tolares, yaretales y bofedales (para la ecorregión puna).

\section{Ecorregión Desierto Costero.}

De las lomas costeras se han hallado hasta cuatro de los ocho tipos clasificados por Koepcke, siendo : lomas de Cactus (Tacahuay, Sama, Quebrada de burros, Wawakiki, Algarrobal) donde predominan Loxanthocereus sextonianus, Neoraimondia arequipensis y Corryocactus brachypetalus, de Tillandsias (Tacna, Calana) en que domina el paisaje Tillandsia werdermani; de herbáceas (Morro Sama, Pampa Cabeza de Vaca, Tacahuay, Pilcuyo, Alfarillo) en que son comunes Trixis paradoxa, Croton alnifolius, Grindellia glutinosa, Calceolaria sp. y otras más, entre las cuales Ferreyra (1987) destaca a Tropaelum majus, y Zegarra (1994) menciona a herbáceas como Coldenia paronychioides, varias especies de Nolana, Palaua velutina y Hoffmansegia miranda; de floresta arbórea (Tacahuay, Cata-cata, Pilcuyo, Algarrobal, Wawakiki) en que pueden hallarse Caesalpinea spinosa, Schinus molle, Acacia macracantha, Carica candicans, Cytharexylum flexuosum, Marrubium vulgare, y en algunos casos Olea europea, Croton $\mathrm{sp}$, Ficus carica y Schinus molle.

El matorral xerofítico es la única vegetación del desierto que existe entre la orilla marina y los valles fluviales (como pampa Magollo y pampa San Francisco - Calana) y en quebradas secas del interior (como quebrada molles secos). Se encuentran alli: Browningia candelaris, Neoraimondia arequipensis, Haageocereus australis, Grindelia glutinosa, Maytenus octogona, Lycopersicum chilense, Lycopersicum peruvianum, y arbustos 
como Tecoma fulva, Nicotiana glauca, Schinus molle y Pluchea chingoyo.

El monte ribereño está alterado por actividades agrícolas en los valles de Moquegua, Locumba, Sama y Tacna; aún así, se puede encontrar en ellos a algunas especies neotropicaies como Persea americana, Pouteria ovobata, Inga feuellei, Schinus molle, Psidium guajava, Gourliea decorticans, Piptadenia grata y Acacia macracantha. El estrato arbustivo y herbáceo está constituido de Nicotiana glauca, Nicotiana paniculata, Cortaderia sp., Gynerium sagittatum, Tessaria integrifolia, Grindelia glutinosa, Equisetum giganteum, entre otros.

El único humedal costero de la zona es el de Ite, formado a partir de depósitos de material sedimentario de relaves mineros y anegado con filtraciones de agua dulce de una irrigación cercana. La flora vascular de este humedal presenta gran coincidencia con la de los pantanos de Villa (Lima), donde se han hallado también las siguientes especies: Eleocharis sp., Typha dominguensis, Bacopa monnieri, Salicornia fruticosa, Distichlis spicata, Cynodon dactylon, Heliotropum curassavicum, coincidiendo con las observaciones de Pizarro \& Franco (1994) y de Zegarra (1995) para Ite. En cuanto a la flora criptogámica, Pizarro (1995) menciona la predominancia entre las Bacillariophytas de Campylodiscus y Nitszchia, reportada por otros autores para albuferas de Lima, y para los humedales de Mejía (Arequipa).

\section{Ecorregión Serranía Esteparia}

Las formaciones espinosas y secas de esta ecorregión se salpican de varias especies de cactáceas, entre las que son comunes: Corryocactus brevistylus, Oreocereus hendriksenianus, Trichocereus tacnaensis, Corryocactus cuajonense, Opuntia exaltata, Erdisia sp., Lobivia sp., Haageocereus australis y Tephrocactus sphaerica. También abunda Ephedra americana, Lycopersicon sp., Baccharis sp. y Festuca sp.

Las laderas andinas albergan en menor número plantas crasas y en su reemplazo se hallan Baccharis petiolata, Grindellia glutinosa, Xanthium sp., Duniella sp., Lupinus sp., Tagetes sp., Poa sp., Passiflora tripartita, Malvastrum sp., Balbisia sp., Hoffmannseggia sp., Cajophora sp aff. horrida, Mutisia acuminata y Solanum lycopersicoides.
Esta última reportada por Zegarra (1979), en laderas de alrededores de Aricota, entre 2800 a 3100 m.s.n.m.

El estrato arbóreo de los valles andinos de Tarata y Palca está matizado por especies introducidas como Buddleia sp. y Eucalyptus globulus, mientras que las especies nativas mejor representadas son: Cantua candelilla, Psitacanthus cuneifolius, Schinus molle, Mutisia acuminata, Kageneckia sp, Colletia spinossisimus entre otros.

En zonas de valles cálidos como Chucatamani y Borogueña (alrededor de 2000 m.s.n.m.) predominan especies características de pisos más bajos como Acacia macracantha, Browningia candelaris $y$ Tessaria integrifolia.

\section{Ecorregión Puna}

Los bofedales cercanos a cuerpos de agua son los ecosistemas más productivos de la zona andina. En ellos se puede hallar a Distichia muscoide, Oxychloe andina, Calamagrostis sp.y en las cercanías parches de Typha sp., Festuca sp., formando asociaciones vegetales a más de 4000 metros de altitud.

Los bosquetes de queñua son escasos en alturas que superan los 4000 m.s.n.m. y predominan Parastrephia, Festuca y Azorella dando lugar a formacionmes de tolares, pajonales y yaretales respectivamente, aunque hay relictos de Polylepis rugulosa y $P$. tarapacana.

La flora de cuerpos de agua altoandinos está constituida principalmente por algas como Nitella sp. y Chara sp. entre las macrofitas y entre las microscópicas diatomeas y algas verde-azules de los géneros Nitzchia, Synedra, Nostoc y Oscillatoria entre otros. En algunas ocasiones la flora criptogámica de estos cuerpos de agua coinciden en su composición con la de rios y lagunas de zonas de serrania esteparia.

\section{IMPORTANCIA DE LOS ESPACIOS BIOGEOGRÁFICOS}

\section{Como centro de origen de las especies.}

En el sur tenemos algunas zonas que son consideradas centros de origen de especies. El valle de Kisguarani (sur de Arequipa), como centro de diversidad de Psidium guajava en el Perú. Por otro lado, según Rick (1991), vivimos en la zona de distribución de parientes silvestres del tomate Lycopersicon chilense y de la planta ancestral Solanum lycopersicoides, los cuales probablemente 
presentan ecotipos distintos a los colectados más al sur, en el desierto de Antofagasta, por investigadores fitomejoradores. Backeberg (1979) menciona para la costa del sur, a un centro de especiación del grupo de los equinocactus, probablemente se refiere al género Islaya, hallado en la costa sur del Perú.

\section{Especies endémicas.}

Entre los vertebrados se señala como el ave endémica de la zona a Eulidia yarrelli, llamado también "Picaflor de Arica". La distribución por parches de Conirostrum tamarugense también denota cierto tipo de endemismo. Por información de Cracraft (1985), se conoce que algunas aves presentan endemisano en el llamado sub centro peruano andino del oeste cuyo límite sur es Arica. Los peces de aguas continentales presentan especies únicas como: Trichomycterus laucaensis, T. chungarensis, Orestias laucaensis, $O$. parinacotensis y Orestias chungarensis.

Zegarra (1994) menciona la característica endémica del género Nolana para las lomas de Tacna. Brako \& Zarucchi (1993) y Zegarra (1979) destacan que Tillandsia werdermani y Solanum lycopersicoides son endémicos de Tacna. Lo mismo podemos decir de Tecoma fulva y Hoffmannseggia spp. para Moquegua, Tacna y Tarapacá. Haageocereus fascicularis, considerada endémica entre Arica y Mamiña (Iquique) por Hoffmann (1989). Otros cactus considerados endémicos son: Trichocereus tacnaensis y Trichocereus torataensis. Una especie arbórea andina antaño común en Palca: Escallonia angustifolia, es considerada endémica del sur del Perú.

Cano et al (1996) afirman que en la vertiente occidental de los andes del sur y en lomas, viven el $100 \%$ de las especies conocidas de las familias Malesherbiaceae y Velloziaceae, lo que las convierte en endémicas para ésta biorregión. Ferreyra (1987) menciona que Tropaelum majus presenta endemismo en las lomas costeras de Arequipa, Moquegua y Tacna. Para nuestra región biogeográfica, Brako \& Zarucchi (1993) señalan otros endemismos de plantas como Cardenanthus peruvianus y Maleshierba ardens y Maleshierba turbinea.

\section{Localidades tipo.}

Aunque solo destaca su importancia taxonómica, nuestra biorregión muestra ciertas localidades tipo para especies descritas. Un ejemplo es Browningia candelaris descrita por Meyen en 1833 para Arica, algo similar ocurre con Haageocereus fascicularis, con localidad tipo en Chapiquiña (Arica).

Telmatobius fué un género creado por Weigmann en base a hallazgos de este batracio en la sierra de Tacna, en el siglo XIX.

Varios reptiles tienen a algún lugar de Tacna como localidad tipo en el Perú: Microlophus heterolepis y M. quadrivittatus para el desierto costero, y Phrynosaura stolzmanni para la ecoregión Puna de Tacna (Carrillo \& Icochea, 1995). Según observaciones realizadas por herpetólogos chilenos, M. quadrivittatus ha sido encontrado de forma aislada en Iquique e llo.

El ratón de Darwin, descrito después de una colecta en Antofagasta el siglo pasado, tiene una subespecie: Phyllotis darwini osgoodi con localidad tipo en Parinacota (Arica).

Recientemente, Wilson (1990) describió una nueva especie de decápodo abisal Paralomis otsusae extraido del fondo marino (1200-1800 metros de profundidad), en un estudio batimétrico realizado frente a las costas de Arica e Iquique.

\section{PROPOSICIONES}

Después de revisar este abultado conjunto de datos proponemos:

- Que el extremo sur se considere un territorio particular de la ecorregión del desierto costero y serranía esteparia con dos subdivisiones: desierto bajo, hasta $1500 \mathrm{msnm}$ (entre $17^{\circ}$ y $19^{\circ} \mathrm{L} . \mathrm{S}$. aproximadamente) y desierto alto, entre 1500 hasta 2200 msnm (presente en algunas zonas del extremo sur y desierto de Atacama).

- Que, vistas las características de endemismos, localidades tipo, centros de origen de especies y límites de distribución de las especies se protejan ecosistemas frágiles dentro de la biorregión como muestras representativas de la biodiversidad regional.

- Quela biorregión sirva de base para la planificación del desarrollo económico social de Tacna, Moquegua, sur occidente de Puno y Arequipa, zonas aledañas del Departamento de La Paz (Bolivia) y I Región de Chile, por presentar las mismas características biogeográficas. 


\section{REFERENCIASBIBLIOGRÁFICAS}

BACKEBERG, V. C. 1979. Das Kakteenlexikon. GustavFischerverlag, Stuttgart.

BELLIDO, E. 1990. Zonas morfológicas del territorio peruano. Bol. Soc. Geog. Lima. VOL. 104 : 6.

BRACK-EGG, A. 1985. Las ecorregiones del Perú. Boletín de Lima VIII(44):17-28. Lima.

BRAKO, L. \& J. ZARUCCHI. 1993. Catálogo de las angiospermas y Gymnospermas del Perú. Missouri Botanical Garden, St. Louis.

CABRERA A. \& A. WILLINK. 1980. Biogeografía de américa latina, Segunda edición. Monografia No.13PDCT, OEA, Washington.

CARRILLO, N. y J. ICOCHEA. 1995. Lista taxonómica preliminar de los reptiles vivientes del Perú. Pub. MHN UNMSM (A)49:1-27, Lima.

CANO, A.; K. YOUNG \& B. LEON. 1996. Areas importantes para la conservación de fanerógamas en el Perú. En: Diversidad Biológica del Perú, L. Rodriguez (ed.). FANPE-GTZ-INRENA, Lima.

CRACRAFT, J. 1985. Historial Biogeography and Patterns of differentiation within the south american avifauna. A.O.U. Otnithological Monographs (36): 49-84.

CHOQUE, E. 1984 La Burguesia Comercial en Tacna. Pako Yunque ed. , Tacna

DAGNINO, V. 1903. Geografía Fisica del Departamento de Tacna. Arica.

FERREYRA, R. 1987. Plantas que el Perú dió al mundo. En : Cinco conferencias, Com. Per. del V Cent. del Descubr. de América, Lima.

FJELDSA, J \& M. KESSLER. 1996. Conserving the biological diversity of Polylepis woodlands of the highlans of Perú and Bolivia. NORDECO, Copenhagen.

FRANCO, J.; ACLETO, C.; CHAMBILLA, V. \& L. SULCA. 1995. Estudio de las algas Rhodophyta del litoral de Tacna. Rev. Ciencia \& Desarrolio (1):5-11. COIN-UNJBG, Tacna.

GLADE, A. y E. NUÑEZ. 1983. Resúmen de antecedentes de flora y fauna de la I región de Chile. Publ. Divulgación 13(1). CONAF, Santiago.

GOMEZ, O. 1992. Revista Nueva Imagen. (2):30.

GOMEZ-MOLINA, E. \& A. LITTLE. 1981. Geoecology of the andes: the natural science basis for research planning. Mountain research and development (1):115-144.

HOFFMANN, A. 1989. Los cactus en la flora silvestre de Chile. Fund. C. Gay-El Mercurio, Santiago.

INRENA, 1994. Diagnóstico del sistema nacional de áreas protegidas (versión preliminar). Proyecto de Coop. Tec. Perú-Alemania. INRENA, Lima.

KALIN, M.; C. VILLAGRAN; C. MARTICORENA \& J. ARMESTO. 1982. Flora y relaciones biogeográficas en los andes del norte de Chile. En: El ambiente natural y las poblaciones humanas de los andes del norte de Chile. Vol. I. MAB-ORCYT, Montevideo.
MACARTHUR, R. 1972. Geographical ecology. Harper \& Row. N.Y.

MARQUEZ, R. 1990. Sea Turtles of the world. FAO Catalogue species catalogue Vol. 11. Rome.

MORENO, T. y N. CORONEL. 1987. Estudio de posibilidad de introducción de especies no nativas. Convenio UNJBGMicroregión Tarata.

MORRIS, M. \& O. PANTY. 1994. Regiones geográficas de Tacna. V Congr. Nac. Multidisc. de Geografía. UNP/Soc. Geog. Lima,

MORRISON, R.; R. ROSS; C. DOWNES and V. PULIDO. 1990. Perú: Atlas of Nearctic shorebirds on the coast of southamerica. Vol. 2.

MOPU. 1990. Desarrollo y medio ambiente en América latina y el caribe. Una visión evolutiva. PNUMA, MOPU, AECI, Madrid.

ONERN, 1986. Perfil ambiental del Perú. ONERN-AID, Lima.

PEARSON, O\&C. PEARSON. 1978. The diversity and abundance of vertebrates along an altitudinal gradient in Perú. Pub. Museo Historia Natural Javier Prado No. 18. UNMSM, Lima.

PIZARRO, J. 1997. Problemas de conservación de recursos naturales de Tacna. I Foro sobre conservación de los recursos naturales de Tacna. CEIMA-COIN UNJBG, Tacna.

PIZARRO, J. y J. FRANCO. 1994. Contribución al conocimiento de la avifauna de los humedales de ite - Tacna. Libro de resúmenes VIII Congr. Iber. de Zool. de vertebrados, Piura.

RICK, Ch. 1991. Recursos genéticos de Tomate en Suramerica revelan verdaderos tesoros. Diversity 7(1-2):60-63. IBPGR.

RIDGELY, R. \& G. TUDOR. 1989 y 1994. The Birds of Southamerica (T. I \& II). Oxford Univ. Press, Tokyo, Oxford.

VAN WAEREBEEK, K.; REYES, J. \& A. LUSCOMBE. 1988. Revisión de Distribución de pequeños cetáceos frente al Perú. Bol. extr. IMARPE. Callao.

VUILLEUMIER, F. 1981. The origin of the High Andean Birds. Natural History Magazine (7):50-57

WILSON, R. 1990. Paralomis otsusae, a new species of Decapoda Anomura from deep water of the chilean coast. Crustaceana 58(2): 130-135.

ZEGARRA, R. 1979. Solanum lycopersicoides Dun., una especie endémica del desierto tacneño. Libro de resúmenes VI Congr. Nac. Biología, Chiclayo.

ZEGARRA, R. 1994. La vegetación desértica perenne de Tacna: estudio biosistemático y sus recursos naturales. Rev. Nueva Imagen (5):53-66. UNJBG, Tacna.

ZEGARRA, R. 1995. La vegetación pantanosa de Ite. Rev. Ciencia \& Desarrollo (1):12-15. COIN-UNJBG, Tacna.

SOUTH AMERICA LAND COVER CHARACTERISTICS DATABASE. 1998. http://www.cr.usgs.gov/landdaac/glcc/ sadoc1_2.html.

GIDDINGS, L. 1992. Zonas aparentes de la vegetación de Sudamerica vistas desde satélites metereológicas. Revista Interciencia (4):112. 
